siderable amount of kidney structure still remained, but it was tough, bleached white, and partly excavated or eroded by ulcerative degeneration. A black stone, of conical shape, measuring about an inch in length, weighing very light, but roughened on its surface by secondary urate deposits, was found in the pelvis. Right kidney and ureter tolerably natural; hypertrophied, but presenting appearances of recent acute, cloudy swelling, or parenchymatous nephritis. Bladder-coats much hypertrophied, contracted, and empty.

\section{WEST RIDING ASYLUM.}

\section{PUERPERAL MANIA; PELVIC HAMATOCELE; SUDDEN DEATH.}

(Under the care of Dr. Crichton Browne.)

THE following case, the clinical notes of which have been taken by Dr. Herbert C. Major and Dr. Frederick Newcombe, illustrates what is believed to be a hitherto unreco. gnised danger in the course of puerperal mania, and an immensely rapid fatality attending hæmorrhage in the vicinity of the uterus.

Frances C-n married, thirty-one years of age, admitted from Leeds on December 15th, 1873, into No. 25 ward.

History.-This is the patient's second attack of insanity. The first occurred five years ago, after the birth of hex fourth child. It was, however, only of a week's duration, and was so mild in character that removal to an asylum was not necessary. Three weeks since she was delivered of her ninth child. The labour is said to have been severe, and to have been followed by considerable flooding. Instruments were not used. After the labour she continued fairly well for ten days, and then symptoms of mental disturbance showed themselves. She became restless, excited, and incoherent in her conversation, raving chiefly on religious topics. Up to the time of her removal to this asylum she has continued to be greatly excited and violent. She has had no sleep, but has taken a fair amount of nourishment The patient's sister died in an asylum when she was under treatment for melancholia; a maternal aunt was also insane. There is no history of intemperance or cranial injury.

On admission she was extremely restless, and talked incessantly, and so rapidly that it was impossible to make out what she said. "Heaven," "hell," and "spirits," however, mingled largely in her discourse. When her attention was arrested by any powerful impression made upon it, she could understand what was said to her, and answered correctly, but the question asked was instantly converted into a fresh starting-point for her incoherent wandering. She was not destructive, never combative, and although in constant movement, did not attempt to get out of bed. Physically she was above the average height, had dark-brown hair, brown eyes, and a rather pale, sallow complezion. She was well nourished. The pupils were normal, the breasts flaccid and empty; the impulse of the heart was in the proper position, but its action was somewhat tumultuous; the pulse was 116, and weak in character; the tongue was dry, and coated with a dirty-yellow fur, and the bowels were confined, but the appetite was good. The urine was free from albumen, and there was no discharge from the vagina. The case was evidently one of puerperal mania, of which the prognosis was apparently favourable. Ordered a black draught at once, and to have extra diet with six ounces of port wine daily. To remain in bed and to have soda-water to drink, and a warm bath each evening.

Dec. 17th. - Still much excited; has had little or no sleep; to take fifteen grains of chloral hydrate night and morning.

18th.- Slept well last night after her chloral draught. Is calmer this morning.

19th.-Did not sleep last night, but is decidedly quieter. Takes food freely.

21st.-Is now quiet and composed for the greater part of the day, with occasional outbursts of talkative excitement.

25th.-Quiet and convalescent; treatment still continued. 26th.-At seven o'clock this evening the patient, who had been perfectly rational and tranquil throughout the day, was in the act of getting out of bed to take her usual warm bath, when she suddenly cried out to the nurse, who was beside her, that she was in pain, and fell back upon the bed looking pale and faint. The medical officer, who was at once summoned, and who was on the spot in less than five minutes, found her struggling to rise but unable to do so, deadly pale, and with no perceptible pulse. She had an anxious expression of countenance, and stared wildly around her, but was unable to answer any questions or even to speak. She was breathing freely. She was at once placed in a recumbent position, while warm bottles were applied to the feet and sinapisms to the præcordia. Brandy, ammonia, and tincture of digitalis were administered by the mouth. Under this treatment distinct improvement took place. In twenty minutes the pulse could be felt at the wist, and consciousness returned; the patient then complained of agonising pains in her knees, and clutched at her heart as if experiencing embarrassment there. A new difficulty, however, now intervened as the delirium of cerebral anæmia came on, and manifested itself in violent struggles, loud screams, and incoherent sentences. The pupils were dilated and the lips quite white. The pulse began to fail, and in half an hour could scarcely be felt at the wrist; the extremities became cold. Brandy and ammonia having been again administered by the mouth and rectum without effect, and clutchings at the breast still continuing, an inhalation of nitrite of amyl was tried. This did not produce its usual physiological effects. There was no trace of flushing, and the pulse, instead of rising, fell under its influence, descending from 130 to 115 . When the inhalation was discontinued the pulse again rose. The sinapisms, though kept long applied, produced no redness. At $8.15 \mathrm{P}$ ir. there was total unconsciousness, and the breatbing became slower. Currents of electricity were sent through the heart, but without any result. Death took place at nine o'clock.

Autopsy, fifty.five hours after death. - Weather cold. The body is well nourished, and is free from bruises and marks. The skin and mucous membranes are extremely pale. Rigor mortis is present both in the arms and legs, and a slight degree of hypostatic congestion is visible on the back. The sinuses of the dura mater are quite empty. The brain is typically healthy in every respect, except that it is very anæmic; it weighs $48 \mathrm{oz}$. There is no fluid in the pericardium, and the heart, which weighs $11 \mathrm{oz}$, is healthy; its cavities are all empty. The lungs are pale, but healthy. On opening the abdomen, a large mass of dark clotted blood is found occupying its lower part. It is extra-peritoneal, and is separated from the abdominal viscera by the peritoneal layer, which remains entire and confines it posteriorly. It extends up to within an inch of the umbilicus, and fully occupies the hypogastric and inguinal regions. At its upper margin it is about an inch in depth, and becomes thicker as it descends into the pelvis, where it seems to surround all the viscera. Behind the clot, and altogether distinct from it, the uterus is found subinvoluted, and of the size of a large orange. Its walls are three-quarters of an inch thick, and its cavity contains no blood. In the fold of the broad ligament, on the left side, a large quantity of clotted blood is discovered, and here, indeed, the clot seems to have had its origin from the uterine veins or the internal iliac veins, which they join. From this point the clot, which has the consistence and appearance of black-currant jelly, extends round the extra-peritoneal parts of the rectum, vagina, and bladder, and then behind the pubis up into the abdomen. (Twenty-five ounces of coagulated blood were removed from the abdomen and pelvis.) All the arteries ar'e entire and healthy. There is a considerable amount of rather hard fæces in the rectum and sigmoid flexure.

Employment of Metalite Mercury. - Dr. Kirchstein relates a successful case of the above in the Berlin Klin. Wochenschrift, No. 35, 1873. The patient was a robust fisherman, subject to cramps in the stomach. There was complete obstruction of the intestinal canal, with tympanites, fæcaloid vomiting, \&c. All the usual external and internal means proved unavailing. After five days, and when the prognosis became most grave, Dr. Kirchstein thought of metallic mercury, and two tablespoonfuls, at half an hour's interval, were administered in the evening. The vomiting soon ceased, and towards the morning the patient passed a great many hard, charcoal-like scybala. He rapidly recovered. During the three weeks following evacuation of globules of mercury was observed. 